\title{
On Some Novel Consequences of Clifford Space Relativity Theory
}

\author{
Carlos Castro \\ November, 2014 \\ Quantum Gravity Research Group, Topanga, CA. 90290 ; Center for Theoretical Studies \\ of Physical Systems, Clark Atlanta University, Atlanta, GA. 30314; \\ perelmanc@hotmail.com

\begin{abstract}
Some of the novel physical consequences of the Extended Relativity Theory in $C$-spaces (Clifford spaces) are presented. In particular, generalized photon dispersion relations which allow for energy-dependent speeds of propagation while still retaining the Lorentz symmetry in ordinary spacetimes, while breaking the extended Lorentz symmetry in $C$-spaces. We analyze in further detail the extended Lorentz transformations in Clifford Space and their physical implications. Based on the notion of "extended events" one finds a very different physical explanation of the phenomenon of "relativity of locality" than the one described by the Doubly Special Relativity (DSR) framework. We finalize with a discussion of the modified dispersion relations, rainbow metrics and generalized uncertainty relations in $C$-spaces which are extensions of the stringy uncertainty relations.
\end{abstract}

Keywords : Clifford algebras; Extended Relativity in Clifford Spaces; Modified Dispersion Relations ; Rainbow Metrics; Generalized Uncertainty Principle.

In the past years, the Extended Relativity Theory in $C$-spaces (Clifford spaces) and Clifford-Phase spaces were developed [1], [2]. The Extended Relativity theory in Cliffordspaces (C-spaces) is a natural extension of the ordinary Relativity theory whose generalized coordinates are Clifford polyvector-valued quantities which incorporate the lines, areas, volumes, and hyper-volumes degrees of freedom associated with the collective dynamics of particles, strings, membranes, p-branes (closed p-branes) moving in a D-dimensional target spacetime background. C-space Relativity permits to study the dynamics of all (closed) p-branes, for different values of $\mathrm{p}$, on a unified footing.

Our theory has 2 fundamental parameters : the speed of a light $c$ and a length scale which can be set equal to the Planck length. The role of "photons" in $C$-space is played by tensionless branes. An extensive review of the Extended Relativity Theory in Clifford 
spaces can be found in [1]. The polyvector valued coordinates $x^{\mu}, x^{\mu_{1} \mu_{2}}, x^{\mu_{1} \mu_{2} \mu_{3}}, \ldots$ are now linked to the basis vectors generators $\gamma^{\mu}$, bi-vectors generators $\gamma_{\mu} \wedge \gamma_{\nu}$, tri-vectors generators $\gamma_{\mu_{1}} \wedge \gamma_{\mu_{2}} \wedge \gamma_{\mu_{3}}$, ... of the Clifford algebra, including the Clifford algebra unit element (associated to a scalar coordinate).

These polyvector valued coordinates can be interpreted as the quenched-degrees of freedom of an ensemble of $p$-loops associated with the dynamics of closed $p$-branes, for $p=0,1,2, \ldots, D-1$, embedded in a target $D$-dimensional spacetime background. $C$-space is parametrized not only by 1 -vector coordinates $x^{\mu}$ but also by the 2 -vector coordinates $x^{\mu \nu}, 3$-vector coordinates $x^{\mu \nu \alpha}, \ldots$, called also holographic coordinates, since they describe the holographic projections of 1-loops, 2-loops, 3-loops,..., onto the coordinate planes . By $p$-loop we mean a closed $p$-brane; in particular, a 1-loop is closed string. When $\mathbf{X}$ is the Clifford-valued coordinate corresponding to the $C l(1,3)$ algebra in four-dimensions it can be decomposed as

$$
\mathbf{X}=s \mathbf{1}+x^{\mu} \gamma_{\mu}+x^{\mu \nu} \gamma_{\mu} \wedge \gamma_{\nu}+x^{\mu \nu \rho} \gamma_{\mu} \wedge \gamma_{\nu} \wedge \gamma_{\rho}+x^{\mu \nu \rho \tau} \gamma_{\mu} \wedge \gamma_{\nu} \wedge \gamma_{\rho} \wedge \gamma_{\tau}
$$

where we have omitted combinatorial numerical factors for convenience in the expansion of eq-(1). To avoid introducing powers of a length parameter $L$ (like the Planck scale $L_{p}$ ), in order to match physical units in the expansion of the polyvector $X$ in eq-(1), we can set it to unity to simplify matters.

The component $s$ is the Clifford scalar component of the polyvector-valued coordinate and $d \Sigma$ is the infinitesimal $C$-space proper "time" interval

$$
(d \Sigma)^{2}=(d s)^{2}+d x_{\mu} d x^{\mu}+d x_{\mu \nu} d x^{\mu \nu}+\ldots
$$

that is invariant under $C l(1,3)$ transformations and which are the Clifford-algebraic extensions of the $S O(1,3)$ Lorentz transformations [1]. One should emphasize that $d \Sigma$ is not equal to the proper time Lorentz-invariant interval $d \tau$ in ordinary spacetime $(d \tau)^{2}=$ $g_{\mu \nu} d x^{\mu} d x^{\nu}=d x_{\mu} d x^{\mu}$. Generalized Lorentz transformations (poly-rotations) in flat $C$ spaces were discussed in [1]. In this work we shall provide an extensive analysis of the $C$-space generalized Lorentz transformations and their physical implications.

Let us provide several examples of generalized Lorentz transformations in $C$-space. For example, given $\gamma_{02}$ the transformation involving the rotor $R_{1}=\cosh (\beta / 2)-\gamma_{02} \sinh (\beta / 2)$ corresponds to an ordinary Lorentz boost transformation along the $X^{2}$ direction and involving the ordinary temporal variable $X^{0}$. The ordinary Lorentz boots generators are given by the bivectors $\gamma_{\mu \nu}$, and which in turn are also expressed as the commutators $\left[\gamma_{\mu}, \gamma_{\nu}\right]$. The physical significance of the latter commutators is that they represent a "rotation" along the $X^{\mu}-X^{\nu}$ directions.

However, since one may also write the bivector $\gamma_{02}$ as the commutator $\left[\gamma_{12}, \gamma_{01}\right]=$ $-2 \gamma_{02}$, the transformation involving the above rotor $R_{1}$ also corresponds to an areal boost along the $X^{12}$ direction but involving the areal temporal coordinate $X^{01}$. Namely, it is a "rotation" along the $X^{12}-X^{01}$ directions. Whereas the ordinary boost is a "rotation" along the $X^{2}-X^{0}$ directions.

After writing 


$$
\left(X^{B}\right)^{\prime} \Gamma_{B}=\left(\cosh (\beta / 2)-\gamma_{02} \sinh (\beta / 2)\right)\left(X^{A} \Gamma_{A}\right)\left(\cosh (\beta / 2)+\gamma_{02} \sinh (\beta / 2)\right)
$$

straightforward algebra yields the transformation of the following bivector coordinates

$$
\begin{aligned}
& \left(X^{12}\right)^{\prime}=X^{12} \cosh \beta+X^{01} \sinh \beta \\
& \left(X^{01}\right)^{\prime}=X^{01} \cosh \beta+X^{12} \sinh \beta
\end{aligned}
$$

One has a mixing of the spatial and temporal areal bivector coordinates in the new frame of reference.

Furthermore, since $\left[\gamma_{013}, \gamma_{123}\right] \sim \gamma_{02}$, the transformation involving the above rotor $R_{1}$ also corresponds to a 3 -volume boost along the $X^{123}$ direction but involving the 3 -volume temporal coordinate $X^{013}$. Namely, it is a "rotation" along the $X^{123}-X^{013}$ directions giving

$$
\begin{aligned}
& \left(X^{123}\right)^{\prime}=X^{123} \cosh \beta+X^{013} \sinh \beta \\
& \left(X^{013}\right)^{\prime}=X^{013} \cosh \beta+X^{123} \sinh \beta
\end{aligned}
$$

One has a mixing of the spatial and temporal trivector coordinates in the new frame of reference. The ordinary Lorentz boosts of the vector coordinates give

$$
\begin{aligned}
& \left(X^{2}\right)^{\prime}=X^{2} \cosh \beta+X^{0} \sinh \beta \\
& \left(X^{0}\right)^{\prime}=X^{0} \cosh \beta+X^{2} \sinh \beta
\end{aligned}
$$

while the remaining coordinates remain invariant and such that the quadratic form $X^{A} X_{A}=\left(X^{A}\right)^{\prime}\left(X_{A}\right)^{\prime}$ remains invariant. Straightforward algebra leads to

$$
\begin{aligned}
& -\left(X_{0}^{\prime}\right)^{2}+\left(X_{1}^{\prime}\right)^{2}-L^{-2}\left(X_{01}^{\prime}\right)^{2}+L^{-2}\left(X_{12}^{\prime}\right)^{2}-L^{-4}\left(X_{013}^{\prime}\right)^{2}+L^{-4}\left(X_{123}^{\prime}\right)^{2}= \\
& -\left(X_{0}\right)^{2}+\left(X_{1}\right)^{2}-L^{-2}\left(X_{01}\right)^{2}+L^{-2}\left(X_{12}\right)^{2}-L^{-4}\left(X_{013}\right)^{2}+L^{-4}\left(X_{123}\right)^{2}
\end{aligned}
$$

The quadratic form is defined as

$$
<\mathbf{X}^{\dagger} \mathbf{X}>=X_{A} X^{A}=s^{2}+X_{\mu} X^{\mu}+X_{\mu_{1} \mu_{2}} X^{\mu_{1} \mu_{2}}+\ldots \ldots X_{\mu_{1} \mu_{2} \ldots \ldots \mu_{D}} X^{\mu_{1} \mu_{2} \ldots \mu_{D}}
$$

where $\mathbf{X}^{\dagger}$ denotes the reversal operation obtained by reversing the order of the gamma generators in the wedge products. The symbol $<\Gamma_{A} \Gamma_{B}>$ denotes taking the scalar part in the Clifford geometric product of $\Gamma_{A} \Gamma_{B}$. It is the analog of the trace of a product of matrices. Such scalar part can be obtained from the (anti) commutator relations of the Clifford algebra generators. For example

$$
\begin{gathered}
<\gamma_{\mu} \gamma^{\nu}>=\delta_{\mu}^{\nu}, \quad<\gamma_{\mu_{1} \mu_{2}} \gamma^{\nu_{1} \nu_{2}}>=-\delta_{\mu_{1} \mu_{2}}^{\nu_{1} \nu_{2}} \\
<\gamma_{\mu_{1} \mu_{2} \mu_{3}} \gamma^{\nu_{1} \nu_{2} \nu_{3}}>=-\delta_{\mu_{1} \mu_{2} \mu_{3} \nu_{2} \nu_{3}}, \quad<\gamma_{\mu_{1} \mu_{2} \mu_{3} \mu_{4}} \gamma^{\nu_{1} \nu_{2} \nu_{3} \nu_{4}}>=\delta_{\mu_{1} \mu_{2} \mu_{3} \mu_{4}}^{\nu_{2} \nu_{3} \nu_{4}}
\end{gathered}
$$


One should note the presence of \pm signs in the right hand side of eqs-(9). They are connected to the even/odd behavior of the reversal operation $\left(\gamma_{C}\right)^{\dagger}= \pm \gamma_{C}$.

The quadratic form is invariant under the isometry transformations

$$
\mathbf{X}^{\prime}=\mathbf{R} \mathbf{X ~ L}^{\dagger}, \quad \mathbf{R}^{\dagger} \mathbf{R}=1, \quad \mathbf{L}^{\dagger} \mathbf{L}=1 \Rightarrow<\mathbf{X}^{\prime \dagger} \mathbf{X}^{\prime}>=<\mathbf{X}^{\dagger} \mathbf{X}>
$$

due to the cyclic property of the scalar part projection

$$
\begin{aligned}
<\mathbf{X}^{\prime \dagger} \mathbf{X}^{\prime}>= & <\mathbf{L} \mathbf{X}^{\dagger} \mathbf{R}^{\dagger} \mathbf{R} \mathbf{X} \mathbf{L}^{\dagger},>=<\mathbf{L X}^{\dagger} \mathbf{X ~ L}^{\dagger}>= \\
& <\mathbf{L}^{\dagger} \mathbf{L} \mathbf{X}^{\dagger} \mathbf{X}=<\mathbf{X}^{\dagger} \mathbf{X}>
\end{aligned}
$$

where $\mathbf{R}, \mathbf{L}$ are Clifford-valued rotors acting on the right and left respectively.

The second example corresponds to the case when there is a mixing of different grades. It involves the commutator $\left[\gamma_{0123}, \gamma_{3}\right] \sim \gamma_{012}$ and such that the transformation involving the rotor $R_{2}=\cosh \left(\beta^{\prime} / 2\right)-\gamma_{012} \sinh \left(\beta^{\prime} / 2\right)$ corresponds to a boost along the spatial $X^{3}$ direction but involving now the temporal 4 -volume polyvector-valued coordinate $X^{0123}$. The reason being that $\gamma_{012}$ can be rewritten as the commutator of $\gamma_{0123}$ and $\gamma_{3}$, so we have now "rotations" along the $X^{3}-X^{0123}$ directions. Straightforward algebra yields now the transformation of the following (poly) vector coordinates

$$
\begin{aligned}
& \left(X^{3}\right)^{\prime}=X^{3} \cosh \left(\beta^{\prime}\right)-L^{-3} X^{0123} \sinh \left(\beta^{\prime}\right) \\
& \left(X^{0123}\right)^{\prime}=X^{0123} \cosh \left(\beta^{\prime}\right)-L^{3} X^{3} \sinh \left(\beta^{\prime}\right)
\end{aligned}
$$

In this case one has a mixing of polyvector-valued coordinates of different grade. In the new frame of reference the spatial $X^{3}$ coordinate and the temporal 4 -volume coordinate $X^{0123}$ are mixed.

Furthermore, since $\left[\gamma_{03}, \gamma_{123}\right] \sim \gamma_{012}$, the transformation involving the rotor $R_{2}=$ $\cosh \left(\beta^{\prime} / 2\right)-\gamma_{012} \sinh \left(\beta^{\prime} / 2\right)$ also corresponds to a boost along the spatial trivector $X^{123}$ direction but involving now the temporal bivector coordinate $X^{03}$. These transformations are

$$
\begin{gathered}
\left(X^{123}\right)^{\prime}=X^{123} \cosh \left(\beta^{\prime}\right)-L X^{03} \sinh \left(\beta^{\prime}\right) \\
\left(X^{03}\right)^{\prime}=X^{03} \cosh \left(\beta^{\prime}\right)-L^{-1} X^{123} \sinh \left(\beta^{\prime}\right)
\end{gathered}
$$

In the above equations we have used the relations

$$
\begin{gathered}
\gamma_{01}^{2}=1, \quad \gamma_{02}^{\dagger}=-\gamma_{02}, \quad \gamma_{012}^{2}=1, \quad \gamma_{012}^{\dagger}=-\gamma_{012} \\
\left\{\gamma_{12}, \gamma_{02}\right\}=0, \quad\left[\gamma_{0123}, \gamma_{012}\right]=-2 \gamma_{3}, \quad\left\{\gamma_{0123}, \gamma_{012}\right\}=0 \\
\gamma_{02} \gamma_{12} \gamma_{02}=-\gamma_{12}, \quad\left[\gamma_{012}, \gamma_{3}\right]=2 \gamma_{0123}, \quad\left\{\gamma_{012}, \gamma_{3}\right\}=0, \ldots
\end{gathered}
$$


$\cosh ^{2}(\xi)-\sinh ^{2}(\xi)=1, \cosh ^{2}(\xi)+\sinh ^{2}(\xi)=\cosh (2 \xi), \sinh (2 \xi)=2 \sinh (\xi) \cosh (\xi)$

Given in general a transformation of the form

$$
\left(\cosh (\beta / 2)-\Gamma_{C} \sinh (\beta / 2)\right) X^{A} \Gamma_{A}\left(\cosh (\beta / 2)+\Gamma_{C} \sinh (\beta / 2)\right)=X^{\prime B} \Gamma_{B}
$$

one learns that

$$
\begin{gathered}
X^{\prime B}=X^{B} \cosh ^{2}(\beta / 2)-X^{A} \sinh ^{2}(\beta / 2)<\Gamma_{C} \Gamma_{A} \Gamma_{C} \Gamma^{B}>+ \\
X^{A} \cosh (\beta / 2) \sinh (\beta / 2)<\left[\Gamma_{A}, \Gamma_{C}\right] \Gamma^{B}>
\end{gathered}
$$

The generator $\Gamma_{C}$ of generalized Lorentz boosts is of the form $\left(\gamma_{0 \mu_{1} \mu_{2} \ldots \mu_{n-1}}\right)$ with the provision that under the reversal operation it changes sign

$$
\left(\gamma_{0 \mu_{1} \mu_{2} \ldots \mu_{n-1}}\right)^{\dagger}=-\gamma_{0 \mu_{1} \mu_{2} \ldots \mu_{n-1}}
$$

so that $\mathbf{R R}^{\dagger}=1$. This condition will restrict the values of $n$ to be $n=2,3,6$, ...and obeying

$$
\left(\gamma_{0 \mu_{1} \mu_{2} \ldots \mu_{n-1}}\right)^{2}=1
$$

Generalized spatial rotations don't involve the temporal directions and are generated by $\gamma_{\mu_{1} \mu_{2} \ldots \mu_{m}}$ obeying

$$
\left(\gamma_{\mu_{1} \mu_{2} \ldots \mu_{m}}\right)^{\dagger}=-\gamma_{\mu_{1} \mu_{2} \ldots \mu_{m}}
$$

and

$$
\left(\gamma_{\mu_{1} \mu_{2} \ldots \mu_{m}}\right)^{2}=-1
$$

For instance, a generalized rotation in $D>4$ and generated by $\gamma_{12 \ldots 6}$ involving the parameter $\alpha^{12 \ldots 6}$ yields a rotor whose Taylor series expansion becomes

$$
\mathbf{R}=e^{\alpha^{12 \ldots 6}} \gamma_{12 \ldots .66}=\cos \left(\alpha^{12 \ldots 6}\right)+\gamma_{012 \ldots \ldots 6} \sin \left(\alpha^{12 \ldots 6}\right)
$$

due to the condition $\left(\gamma_{12 \ldots . .6}\right)^{2}=-1$ which is similar to having the imaginary unit $i^{2}=-1$ and the expression $e^{i \theta}=\cos (\theta)+i \sin (\theta)$. For an earlier discussion of generalized rotations within C-space see [6]. Whereas a generalized Lorentz boost is like having a "rotation" with an imaginary "angle" leading to the hyperbolic functions

$$
\mathbf{R}=e^{\beta^{012 \ldots 5} \gamma_{02 \ldots . .5}}=\cosh \left(\beta^{012 \ldots .5}\right)+\gamma_{012 \ldots . .5} \sinh \left(\beta^{12 \ldots 5}\right)
$$

due to the condition $\left(\gamma_{012 \ldots . .5}\right)^{2}=1$.

Eq-(17) only simplifies considerably in the very special case when the values of the polyvector valued indices $A, B, C$ are such that

$$
<\Gamma_{C} \Gamma_{A} \Gamma_{C} \Gamma^{B}>=-1, \quad<\left[\Gamma_{A}, \Gamma_{C}\right] \Gamma^{B}>= \pm 2
$$


and it leads to the type of transformations displayed above. In general, for a given set of values of $B, C$, one must sum over all the $A$ indices in eq-(17). For this reason the most general expression for $X^{\prime B}$ given by eq-(17) is more complicated than that given by the above equations. Another special case occurs when

$$
<\Gamma_{C} \Gamma_{A} \Gamma_{C} \Gamma^{B}>=1, \quad<\left[\Gamma_{A}, \Gamma_{C}\right] \Gamma^{B}>=0
$$

leading to $X^{B}=X^{B}$ so that these particular polyvector coordinate components remain invariant.

One should emphasize that the functional form of the most general transformations are even more complicated than those described in eq-(17). Let us write the rotor associated with a "rotation" along the $X^{A}-X^{B}$ directions in $C$-space with parameter $\alpha^{A B}$, after writing the commutation relations $\left[\Gamma_{A}, \Gamma_{B}\right]=f_{A B} \Gamma_{C}$, as follows

$$
\mathbf{R}=e^{\alpha^{A B}\left[\Gamma_{A}, \Gamma_{B}\right]}=e^{\alpha^{A B}} f_{A B}^{C} \Gamma_{C}=e^{\beta^{C} \Gamma_{C}}, \beta^{C}=\alpha^{A B} f_{A B}{ }^{C}
$$

where $f_{A B} C$ are the structure constants of the algebra. There is a summation over the $C$ indices (but not over the $A, B$ indices) in eq-(25) and the reversal condition reads

$$
\left[\Gamma_{A}, \Gamma_{B}\right]^{\dagger}=-\left[\Gamma_{A}, \Gamma_{B}\right] \Rightarrow \mathbf{R} \mathbf{R}^{\dagger}=1
$$

and which is satisfied in particular when $\Gamma_{A}^{\dagger}=-\Gamma_{A} ; \Gamma_{B}^{\dagger}=-\Gamma_{B}$ giving $\Gamma_{C}^{\dagger}=-\Gamma_{C}$. This is a result of the relations $\left(\Gamma_{A} \Gamma_{B}\right)^{\dagger}=\left(\Gamma_{B}\right)^{\dagger}\left(\Gamma_{A}\right)^{\dagger}=\Gamma_{B} \Gamma_{A}$. In the most general case, for arbitrary dimensions, due to the summation over the $C$ polyvector indices in eq- $(25)$, the rotor $\mathbf{R}$ cannot be expressed in the form displayed in eq-(16) after performing a Taylor series expansion of the exponentials. For instance

$$
e^{\beta^{01} \gamma_{01}+\beta^{023} \gamma_{023}} \neq\left(\cosh \left(\beta^{01}\right)+\gamma_{01} \sinh \left(\beta^{01}\right)\right)\left(\cosh \left(\beta^{023}\right)+\gamma_{023} \sinh \left(\beta^{023}\right)\right)
$$

as a result of the Baker-Campbell-Hausdorf formula. Because $\left[\gamma_{01}, \gamma_{023}\right] \neq 0$ the left hand side of eq-(27) does not factorize.

We learnt from Special Relativity that the concept of simultaneity is relative. The typical example arises when a moving observer inside a train sees the front and back doors of a train opening simultaneously. Due to the spatial separation $\left(\Delta X^{3} \neq 0\right)$ between the two doors, an observer at rest in the platform will see the doors opening at different times

$$
\left(\Delta X^{0}\right)^{\prime}=\Delta X^{0} \cosh (\beta)+\Delta X^{3} \sinh (\beta) \neq 0
$$

despite $\Delta X^{0}=0$ due to the fact that $\Delta X^{3} \neq 0$.

Something analogous, and more general, occurs in $C$-space. Let us denote by $\Delta X^{3}=$ $X_{(2)}^{3}-X_{(1)}^{3}, \Delta X^{0123}=X_{(2)}^{0123}-X_{(1)}^{0123}$ the spatial and 4-volume separation, respectively, between two events $\mathbf{1}$ and $\mathbf{2}$ in a given frame of reference in a flat $C$-space. From eqs-(12) it follows that in the new frame of reference one has

$$
\left(\Delta X^{3}\right)^{\prime}=\Delta X^{3} \cosh \left(\beta^{\prime}\right)-L^{-3} \Delta X^{0123} \sinh \left(\beta^{\prime}\right)
$$




$$
\left(\Delta X^{0123}\right)^{\prime}=\Delta X^{0123} \cosh \left(\beta^{\prime}\right)-L^{3} \Delta X^{3} \sinh \left(\beta^{\prime}\right)
$$

if $\Delta X^{0123} \neq 0$ one has that $\left(\Delta X^{3}\right)^{\prime} \neq 0$ despite that $\Delta X^{3}=0$. Therefore, because $\left(\Delta X^{3}\right)^{\prime} \neq 0$ the observer in the new frame of reference does not experience events $\mathbf{1}, 2$ at the same location.

An "extended" event in $C$-space described by eqs-(29) can be envisaged as follows. An observer assigns to a physical event the coordinate values $X^{A}$ where the index $A$ spans $2^{D}$ values corresponding to the dimension of a Clifford algebra in $D$-dim. In particular $X^{3}, X^{0123}$. Event 1 can be described in terms of a spherical bubble (a closed 3-brane) moving in spacetime whose 4 -volume (swept by the 3 -brane at a given time $X_{(1)}^{0}$ ) is given by $X_{(1)}^{0123}$. The center of mass of such bubble is given by the $X_{(1)}^{\mu}$ coordinates, in particular $X_{(1)}^{3}$ represents the $z$-component. Whereas event $\mathbf{2}$ is described in terms of another spherical bubble of different size in spacetime whose 4-volume at a given time $X_{(2)}^{0}$ is given by $X_{(2)}^{0123}$. The center of mass of such bubble is given now by $X_{(2)}^{\mu}$ coordinates, in particular $X_{(2)}^{3}$. If the centers of mass of the small and large bubble coincide one has that $\Delta X^{3}=0$, while $\Delta X^{0123} \neq 0$ since the bubbles are of different size. Consequently one learns from eq-(29a) that $\left(\Delta X^{3}\right)^{\prime} \neq 0$ in the new frame of reference : namely, the centers of mass of the bubbles in the new frame of reference do no longer coincide.

Concluding, the concept of spacetime locality is relative due to the mixing of 4-volume coordinates with spacetime vector coordinates under generalized Lorentz transformations in $C$-space. In the most general case, there will be mixing of all polyvector valued coordinates. This was the motivation to build a unified theory of all extended objects, $p$-branes, for all values of $p$ subject to the condition $p+1=D$. Therefore, the Extended Relativity Theory in $C$-spaces (Clifford spaces) were provides a very different physical explanation of the phenomenon of "relativity of locality" than the one described by the Doubly Special Relativity (DSR) framework [7].

Next we will show how the quadratic Casimir invariant in $C$-space leads to modified wave equations, dispersion laws and to the generalizations of the stringy-uncertainty principle relations. The on-shell mass condition for a massless polyparticle in the $2^{4}$ dimensional $C$-space corresponding to a Clifford algebra in $D=4$, can be rewritten in terms of the polyvector valued components of a wave polyvector $\mathbf{K}$, after setting $L=1, \hbar=c=1$ for simplicity, as

$$
k^{2}+K_{\mu} K^{\mu}+K_{\mu_{1} \mu_{2}} K^{\mu_{1} \mu_{2}}+\ldots \ldots+K_{\mu_{1} \mu_{2} \ldots \mu_{4}} K^{\mu_{1} \mu_{2} \ldots \mu_{4}}=\mathcal{M}^{2}=0
$$

A particular slice through the $2^{4}$-dimensional $C$-space can be taken by imposing the set of algebraic conditions

$$
\begin{gathered}
k^{2}=0, \quad K_{\mu_{1} \mu_{2}} K^{\mu_{1} \mu_{2}}=\lambda_{1}\left(K_{\mu} K^{\mu}\right)^{2}=\lambda_{1} K^{4} \\
K_{\mu_{1} \mu_{2} \mu_{3}} K^{\mu_{1} \mu_{2} \mu_{3}}=\lambda_{2}\left(K_{\mu} K^{\mu}\right)^{3}=\lambda_{2} K^{6}, K_{\mu_{1} \mu_{2} \mu_{3} \mu_{4}} K^{\mu_{1} \mu_{2} \mu_{3} \mu_{4}}=\lambda_{3}\left(K_{\mu} K^{\mu}\right)^{4}=\lambda_{3} K^{8}
\end{gathered}
$$


where the $\lambda$ 's are numerical parameters. Since $k$ is the Clifford scalar part of the wave polyvector it is invariant under $C$-space transformations. Hence the condition $k^{2}=0$ will not break the $C$-space symmetry. However the other slice conditions in eqs-(31a, $31 b)$ will break the generalized (extended) Lorentz symmetry in $C$-space because these conditions are not preserved under the most general $C$-space transformations as described earlier. There will be only the residual standard Lorentz symmetry (in ordinary spacetime) remaining which preserves these conditions/constraints in eqs-(31a, 31b).

Inserting the conditions of eqs-(31) into eq-(30), after setting $k^{2}=0$, yields the modified dispersion law

$$
K^{2}\left(1+\lambda_{1} K^{2}+\lambda_{2} K^{4}+\lambda_{3} K^{6}\right)=\mathcal{M}^{2}-k^{2}=0
$$

Upon writing explicitly

$$
K^{2}=K_{\mu} K^{\mu}=|\vec{K}|^{2}-\left(K_{0}\right)^{2}=|\vec{K}|^{2}-(\omega)^{2}
$$

and solving the algebraic equation for $\omega$ in terms of $|\vec{K}|$ obtained from eq-(32) leads to $\omega=\omega(|\vec{K}|)$. Finally, the group velocity (after reinstating $c$ ) is given by

$$
c(|\vec{K}|)=\frac{\partial \omega(|\vec{K}|)}{\partial|\vec{K}|}=c+\ldots
$$

The group velocity might be greater, smaller or equal to $c$. From eq-(32) one can deduce immediately that one solution is $K^{2}=|\vec{K}|^{2}-(\omega)^{2}=0 \Rightarrow \omega=|\vec{K}| \Rightarrow \frac{\partial \omega(|\vec{K}|)}{\partial|\vec{K}|}=1$ (in $c=1$ units) and as expected massless particles move at the speed of light. However, there are other solutions to eq-(32) besides the trivial one leading to energy dependent speed of propagation. Setting $K^{2}=Z$ leads to a cubic equation inside the parenthesis of eq- $(32)$

$$
1+\lambda_{1} Z+\lambda_{2} Z^{2}+\lambda_{3} Z^{3}=0
$$

that can be solved exactly in terms of the $\lambda$ 's parameters giving $3 \operatorname{roots} z_{i}\left(\lambda_{1}, \lambda_{2}, \lambda_{3}\right), i=$ $1,2,3$. The roots can be all real, or one real and a pair of complex conjugate roots. In the former case we have (after reinstating $c$ and adjusting the proper units for $z_{i}$ ) the particular solutions are

$$
\begin{gathered}
K^{2}=c^{2}|\vec{K}|^{2}-(\omega)^{2}=z_{i}\left(\lambda_{1}, \lambda_{2}, \lambda_{3}\right), \quad \Rightarrow \omega=\sqrt{c^{2}|\vec{K}|^{2}-z_{i}} \Rightarrow \\
c(|\vec{K}|)=\frac{\partial \omega(|\vec{K}|)}{\partial|\vec{K}|}=c \frac{c|\vec{K}|}{\sqrt{c^{2}|\vec{K}|^{2}-z_{i}}}=c \frac{\sqrt{(\omega)^{2}+z_{i}}}{\omega} i=1,2,3
\end{gathered}
$$

Therefore, from eq-(36) one has an energy dependent speed of propagation that can be superluminal if $z_{i}>0$, or subluminal if $z_{i}<0$, in the case one has 3 real roots to the cubic equation (35). One should add that after differentiating $c^{2}|\vec{K}|^{2}-(\omega)^{2}=z_{i}$ in eq-(36) gives

$$
2 c^{2}|\vec{K}| d|\vec{K}|=2 \omega d \omega \Rightarrow c^{2}=\frac{\omega}{|\vec{K}|} \frac{d \omega}{d|\vec{K}|}
$$


leading always to the standard relation $v_{\text {group }} v_{\text {phase }}=c^{2}$ between group and phase velocities for all the possible solutions. The above results were all obtained by setting the Clifford scalar part $k$ of the wave polyvector to zero. The calculations in the simplest $D=2$ case when $k^{2} \neq 0$ can be found in [5] leading also to the possibility of superluminal propagation.

Thus the key novel results one obtains from our analysis of wave propagation in $C$ space when $k^{2}=0$ are :

1. Irrespective of the solutions found in eqs- $(35,36)$ the standard dispersion relation $K^{2}=c^{2}|\vec{K}|^{2}-(\omega)^{2}=0$ is always a solution to eq-(32) giving a constant speed of photon propagation. This is a valid solution to choose whether or not an energy-dependent photon speed is found.

2 . Because the modified dispersion relation in eq-(32) is Lorentz invariant since the proper norm $K^{2}=c^{2}|\vec{K}|^{2}-(\omega)^{2}$ is Lorentz invariant, one is able to arrive at the energy-dependent speed of propagation $c(|\vec{K}|)$ in eqs-(36) while still retaining the Lorentz symmetry. This does not occur in DSR nor in other approaches.

The on-shell mass condition for a massive polyparticle moving in the $2^{4}$-dimensional flat $C$-space, corresponding to a Clifford algebra in $D=4$, can be written in terms of the polymomentum (polyvector-valued) components, in natural units $L=L_{P}=1, \hbar=c=1$, as

$$
\pi^{2}+p_{\mu} p^{\mu}+p_{\mu_{1} \mu_{2}} p^{\mu_{1} \mu_{2}}+p_{\mu_{1} \mu_{2} \mu_{3}} p^{\mu_{1} \mu_{2} \mu_{3}}+p_{\mu_{1} \mu_{2} \ldots \mu_{4}} p^{\mu_{1} \mu_{2} \ldots \mu_{4}}=-\mathcal{M}^{2}
$$

Let us break the ordinary Lorentz invariance by imposing the non-Lorentz invariant conditions on the poly-momenta in $C$-space

$$
\begin{gathered}
p_{i j} p^{i j}=\beta_{1}|\vec{p}|^{4}, \quad p_{i j k} p^{i j k}=\beta_{2}|\vec{p}|^{6} \\
p_{0 i} p^{0 i}=\alpha_{1}\left(p_{0}\right)^{2}|\vec{p}|^{2}, \quad p_{0 i j} p^{0 i j}=\alpha_{2}\left(p_{0}\right)^{2}|\vec{p}|^{4}, \quad p_{0 i j k} p^{0 i j k}=\alpha_{3}\left(p_{0}\right)^{2}|\vec{p}|^{6}
\end{gathered}
$$

where the $\alpha$ 's and $\beta$ 's are numerical parameters. The mass-shell condition in $C$-space $P_{A} P^{A}=-\mathcal{M}^{2}$ becomes after inserting the conditions (39) and taking into account the chosen signature $(-,+,+,+)$

$|\vec{p}|^{2}\left(\frac{\pi^{2}}{|\vec{p}|^{2}}+1+\beta_{1}|\vec{p}|^{2}+\beta_{2}|\vec{p}|^{4}\right)-\left(p_{0}\right)^{2}\left(1+\alpha_{1}|\vec{p}|^{2}+\alpha_{2}|\vec{p}|^{4}+\alpha_{3}|\vec{p}|^{6}\right)=-\mathcal{M}^{2}$

One may notice that the terms inside the parenthesis in eq-(40) behave as if one had a rainbow metric as follows

$$
g^{i j}\left(\pi^{2},|\vec{p}|^{2}\right) p_{i} p_{j}+g^{00}\left(|\vec{p}|^{2}\right) p_{0} p_{0}=g^{2}\left(\pi^{2},|\vec{p}|^{2}\right)|\vec{p}|^{2}-f^{2}\left(|\vec{p}|^{2}\right) E^{2}=-\mathcal{M}^{2}
$$

A rainbow metric [8] is a one-parameter family of metrics which depends on the energy (momentum) of the test particles moving in a given spacetime background, and forming 
a rainbow of metrics (rainbow geometry). Setting $\pi^{2}=0$ in eq-(41) one has then that the squared rainbow functions are given by

$$
\begin{gathered}
g^{2}\left(\pi^{2}=0,|\vec{p}|^{2}\right) \equiv 1+\beta_{1}|\vec{p}|^{2}+\beta_{2}|\vec{p}|^{4}, \quad \beta_{1}, \beta_{2}>0 \\
f^{2}\left(|\vec{p}|^{2}\right) \equiv 1+\alpha_{1}|\vec{p}|^{2}+\alpha_{2}|\vec{p}|^{4}+\alpha_{3}|\vec{p}|^{6}, \quad \alpha_{1}, \alpha_{2}, \alpha_{3}>0
\end{gathered}
$$

Given

$$
\begin{gathered}
g^{i j}=g^{2}\left(\pi^{2}=0,|\vec{p}|^{2}\right) \delta^{i j}=\left(1+\beta_{1}|\vec{p}|^{2}+\beta_{2}|\vec{p}|^{4}\right) \delta^{i j} \\
g^{00}=-f^{2}\left(|\vec{p}|^{2}\right) \delta^{00}=-\left(1+\alpha_{1}|\vec{p}|^{2}+\alpha_{2}|\vec{p}|^{4}+\alpha_{3}|\vec{p}|^{6}\right)
\end{gathered}
$$

the rainbow metric is then defined as

$$
\begin{gathered}
d s^{2}=g_{\mu \nu} d x^{\mu} d x^{\nu}= \\
-\left(1+\alpha_{1}|\vec{p}|^{2}+\alpha_{2}|\vec{p}|^{4}+\alpha_{3}|\vec{p}|^{6}\right)^{-1}(d t)^{2}+\left(1+\beta_{1}|\vec{p}|^{2}+\beta_{2}|\vec{p}|^{4}\right)^{-1}\left(d x^{i}\right)^{2}
\end{gathered}
$$

Another physical consequence is that the rainbow metric (44) when $\alpha_{3}=0 ; \alpha_{1}=\beta_{1} ; \alpha_{2}=$ $\beta_{2}$ yields modifications of the Weyl-Heisenberg algebra

$$
\left[x^{\mu}, p^{\nu}\right]=i \hbar g^{\mu \nu}\left(|\vec{p}|^{2}\right)
$$

resulting from the momentum-dependent metric (44), and which in turn leads to the following uncertainty relations

$$
\Delta x^{\mu} \Delta p^{\nu} \geq \frac{\hbar}{2}\left|<\left(1+\alpha_{1}|\vec{p}|^{2}+\alpha_{2}|\vec{p}|^{4}\right)>\eta^{\mu \nu}\right|
$$

where $<\ldots .>$ denote the QM expectation values $<\Psi|\ldots \ldots| \Psi>$. See [9] for rigorous mathematical details.

From (46) one arrives at the minimal length stringy uncertainty relations [10]

$$
\Delta x \Delta p_{x} \geq \frac{\hbar}{2}\left(1+\alpha_{1}\left(\Delta p_{x}\right)^{2}\right) \Rightarrow \Delta x \geq \frac{\hbar}{2 \Delta p_{x}}+\left(\frac{\hbar \alpha_{1}}{2}\right) \Delta p_{x}
$$

Minimizing the expression in (47) and inserting the Planck scale $L_{P}$ which was set to unity one has for the minimum position uncertainty a quantity of the order of the Planck scale

$$
(\Delta x)_{\min }=L_{P} \sqrt{\alpha_{1}}, \quad \alpha_{1}>0
$$

Higher order corrections to the stringy uncertainty relations in eq-(47) stem from the higher grade polymomentum variables in $C$-space appearing in eq-(46) and correspond, physically, to the membrane contributions to the modified uncertainty relations. Hence, the stringy and membrane corrections to the uncertainty relations in $D=4$ are of the form (similar equations follow for the other spatial coordinates) 


$$
\Delta x \Delta p_{x} \geq \frac{\hbar}{2}\left[1+\alpha_{1}\left(\Delta p_{x}\right)^{2}+\alpha_{2}\left(\Delta p_{x}\right)^{4}\right]
$$

leading to

$$
\Delta x \geq \frac{\hbar}{2}\left[\frac{1}{\Delta p_{x}}+\alpha_{1}\left(\Delta p_{x}\right)+\alpha_{2}\left(\Delta p_{x}\right)^{3}\right]
$$

the extremization problem of (50) is more complicated but there is a local minimum when $\alpha_{1}>0, \alpha_{2}>0$. The value of $\Delta p_{x}$ which yields the local minimum for $\Delta x$ is

$$
\left(\Delta p_{x}\right)_{o}=\left(\frac{-\alpha_{1}+\sqrt{\left(\alpha_{1}\right)^{2}+12 \alpha_{2}}}{6 \alpha_{2}}\right)^{\frac{1}{2}}, \alpha_{1}>0, \alpha_{2}>0
$$

If one sets the above value of $\left(\Delta p_{x}\right)_{o}$ and minimal length uncertainty to coincide with the Planck momentum and Planck scale, respectively, one can fix the numerical values of $\alpha_{1}, \alpha_{2}$. In higher dimensions than $D=4$ one will capture the $p$-brane contributions beyond the membrane case due to the contributions of the higher grade polymomenta components. The dimensions (units) of the parameters in eqs- $(49-51)$ are $\left[\beta_{1}\right]=(L / \hbar)^{2},\left[\beta_{2}\right]=(L / \hbar)^{4}$.

Related to the minimal length uncertainty in eq-(48) one should mention that the theory of Scale Relativity proposed by Nottale [11] is based on a minimal observational length-scale, the Planck scale, as there is in Special Relativity a maximum speed, the speed of light, and deserves to be looked within the Clifford algebraic perspective. In future work we shall address the fractal nature of quantum spacetime [11] within the framework of quantum Clifford algebras and Scale Relativity. In the quantization program of gravity a key role must be played by quantum Clifford-Hopf algebras since the latter $q$-Clifford algebras naturally contain the $\kappa$-deformed Poincare algebras [12], [13], which are essential ingredients in the formulation of DSR within the context of Noncommutative spaces. The Minkowski spacetime quantum Clifford algebra structure associated with the conformal group and the Clifford-Hopf alternative $\kappa$-deformed quantum Poincare algebra was investigated [14].

\section{Acknowledgments}

We are indebted to M. Bowers for assistance and to the organizers of the Clifford algebra conference in Tartu, Estonia, for their kind invitation. We thank also Tony Smith, Klee Irwin, Juan Carlos Zambrano, Roberto Lechter and Mohammed Ardehali for their kind support.

\section{References}

[1] C. Castro and M. Pavsic, "The Extended Relativity Theory in Clifford-spaces", Progress in Physics, vol. 1 ( 2005 ) 31. Phys. Letts B 559 (2003) 74. Int. J. Theor. Phys 42 (2003) 1693. 
[2] C. Castro, "The Extended Relativity Theory in Clifford Phase Spaces and Modifications of Gravity at the Planck/Hubble scales" , to appear in Advances in Applied Clifford Algebras.

[3] M. Pavsic, Found. of Phys. 33 (2003) 1277.

M. Pavsic, "The Landscape of Theoretical Physics : A Global View, from point particles to the brane world and beyond, in search of a Unifying Principle"' , (Fundamental Theories of Physics, vol. 19, Kluwer Academic Publishers, Dordrecht, Boston, London, 2001).

[4] C. Castro, "Novel Physical Consequences of the Extended Relativity in Clifford Spaces", to appear in Advances in Applied Clifford Algebras.

[5] C. Castro, "Superluminal particles and the Extended Relativity Theories", Foundations of Physics vol 42, issue 9 (2012) 1135.

[6] M. Pavsic, J. Phys. A41 :332001, (2008).

[7] G. Amelino-Camelia, Int. J. Mod. Phys D 11 (2002) 35. Int. J. Mod. Phys D 11 (2002) 1643.

G. Amelino-Camelia, L. Freidel, J. Kowalski-Glikman and L. Smolin, "The principle of relative locality" arXiv.org : 1101.0931.

[8] J. Magueijo and L. Smolin, "Gravity's Rainbow", Class. Quant. Grav. 21, 1725$1736(2004)$

[9] A. Kempf and G. Mangano, "Minimal Length Uncertainty Relation and Ultraviolet Regularisation", Phys. Rev. D 55, 7909-7920 (1997).

[10] D. Gross and P. Mende, "The high-energy behavior of string scattering amplitudes", Phys. Lett B 197, 129-134 (1987).

D. Amati, M. Ciafaloni and G. Veneziano, "Superstring collisions at planckian energies", Phys. Lett B 197, 81-88 (1987).

[11] L. Nottale, Scale Relativity And Fractal Space-Time: A New Approach to Unifying Relativity and Quantum Mechanics ( 2011 World Scientific Publishing Company).

L. Nottale, Fractal Space-Time and Micro-physics (World Scientific, May 1993).

[12] J. Lukierski, A. Nowicki, H. Ruegg and V. Tolstoy, "q-deformation of Poincar algebra", Phys. Letts B 264, 331-338 (1991).

[13] S. Majid and H. Ruegg, "Bicrossproduct structure of $\kappa$-Poincare group and noncommutative geometry", Phys. Letts B 334, 348-354 (1994).

[14] R. da Rocha, A. E. Bernardini and J. Vaz Jr, $\kappa$-deformed Poincare Algebras and Quantum CliffordHopf Algebras Int. J. Geom. Meth. Mod. Phys. 7, 821-836 (2010). 